\title{
On the Mechanism of ZDDP Antiwear Film Formation
}

\author{
Jie Zhang ${ }^{1} \cdot$ Hugh Spikes ${ }^{1}$ (i)
}

Received: 15 May 2016/Accepted: 13 June 2016/Published online: 29 June 2016

(c) The Author(s) 2016. This article is published with open access at Springerlink.com

\begin{abstract}
Zinc dialkyldithiophosphate additives are used to control wear and inhibit oxidation in almost all engine oils as well as many other types of lubricant. They limit wear primarily by forming a thick, protective, phosphate glass-based tribofilm on rubbing surfaces. This film formation can occur at low temperatures and is relatively indifferent to the chemical nature of the substrate. There has been considerable debate as to what drives ZDDP tribofilm formation, why it occurs only on surfaces that experience sliding and whether film formation is controlled primarily by temperature, pressure, triboemission or some other factor. This paper describes a novel approach to the problem by studying the formation of ZDDP films in full film EHD conditions from two lubricants having very different EHD friction properties. This shows that ZDDP film formation does not require solid-solid rubbing contact but is driven simply by applied shear stress, in accord with a stress-promoted thermal activation model. The shear stress present in a high-pressure contact can reduce the thermal activation energy for ZDDP by at least half, greatly increasing the reaction rate. This mechanism explains the origins of many practically important features of ZDDP films; their topography, their thickness and the conditions under which they form. The insights that this study provides should prove valuable both in optimising ZDDP structure and in modelling ZDDP antiwear behaviour. The findings also highlight the importance of mechanochemistry to the behaviour of lubricant additives in general.
\end{abstract}

\footnotetext{
Hugh Spikes

h.spikes@imperial.ac.uk

Imperial College London, London, UK
}

Keywords ZDDP · Zinc dialkyldithiophosphate · Antiwear · Tribochemistry · Mechanochemistry · Stresspromoted thermal activation - Activation energy $\cdot$ Reaction rate

\section{Background}

Zinc dialkyl- and diaryldithiophosphates (ZDDPs) having the general structure shown in Fig. 1 have been used as antiwear additives for more than 60 years and are still a critical component of almost all modern gasoline and diesel engine lubricants [1].

These additives have been extensively researched, and it is now recognised that they form quite thick reaction films on rubbing surfaces, consisting primarily of amorphous zinc phosphate [2,3]. The phosphate layer is graded, containing predominantly ortho- and pyro-phosphate close to the substrate but having a large proportion of polyphosphate near the top of the film. ZDDP films are rough, comprised of micron-scale circular or elongated pads which can reach up to $200 \mathrm{~nm}$ thickness, separated by deep valleys, as shown in the AFM image and profile in Fig. 2 [4]. The profile $A B$ is taken along the solid white line in the image while the dashed line marks the edge of the rubbed track. The ZDDP films are believed to control wear by limiting direct contact of the two rubbing substrates, thereby preventing the latters' adhesion and reducing the transient contact stresses they experience during sliding [1]. It has also been suggested that ZDDP may react with hard, abrasive iron oxide wear particles to form softer and thus more benign iron sulphides [5].

At very high temperatures, typically above $150^{\circ} \mathrm{C}$, ZDDPs react slowly to form films on solid surfaces even in the absence of rubbing and such films are termed "thermal 
films" [6]. However, when surfaces rub together in a ZDDP-containing lubricant, ZDDP films are generated rapidly and at much lower temperatures, at least as low as $25{ }^{\circ} \mathrm{C}[6,7]$. These are called "tribofilms". Analysis suggests that the two types of film have similar composition [8]. Figure 3 shows optical interference images of the film formed on a steel ball rubbing against a steel disc in ZDDP solution at $100{ }^{\circ} \mathrm{C}$, illustrating how a ZDDP tribofilm of average thickness $120 \mathrm{~nm}$ is formed only on the rubbed track at this temperature [9].

Most research has studied ZDDP behaviour on ferrous surfaces, but it has been shown that ZDDPs also form both thermal films and tribofilms on a wide range of materials, including other metals [10-13], ceramics [14-16], silicon [17] and DLC coatings [18].

Various suggestions have been made as to what drives the formation of ZDDP tribofilms to cause them to form preferentially on rubbing surfaces. Possible factors include

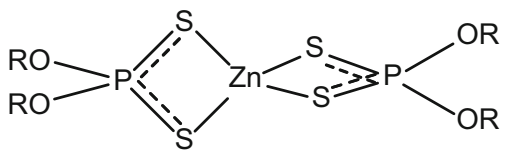

Fig. 1 Molecular structure of ZDDP flash temperature rise, pressure, triboemission and surface catalysis. When solid surfaces are rubbed together, heat is generated which results in a local and transient temperature rise termed "flash temperature" and it has been proposed that this might cause ZDDP tribofilm formation [19]. The magnitude of this temperature rise is governed by moving heat source theory and depends on the rate of heat generation (the product of friction and sliding speed), the speed of the surfaces with respect to the contact, the thermal properties of the solid surfaces and the contact dimensions [20]. Flash temperature rises may be very substantial at high sliding speeds but are generally quite small at sliding speeds below $0.1 \mathrm{~m} / \mathrm{s}$. ZDDPs have been shown to form tribofilms at very low sliding speeds when flash temperature rise is negligible, suggesting that the latter is not a key driver for ZDDP film formation [6]. However, flash temperature calculations are based on the assumption of a Boltzmann energy distribution and it has been suggested that the very intense energy dissipation at asperity conjunctions may lead to non-Boltzmann energy distributions.

It has also been proposed that the very high pressures present in non-conforming contacts such as exist in gears, rolling bearings and the ball-on-flat geometry commonly used in rubbing experiments may promote chemical reaction of ZDDP. Based on quantum chemical simulations, Mosey et al. [21] proposed that very high pressures can
Fig. 2 AFM topography map and profile of ZDDP tribofilm; adapted from [4]
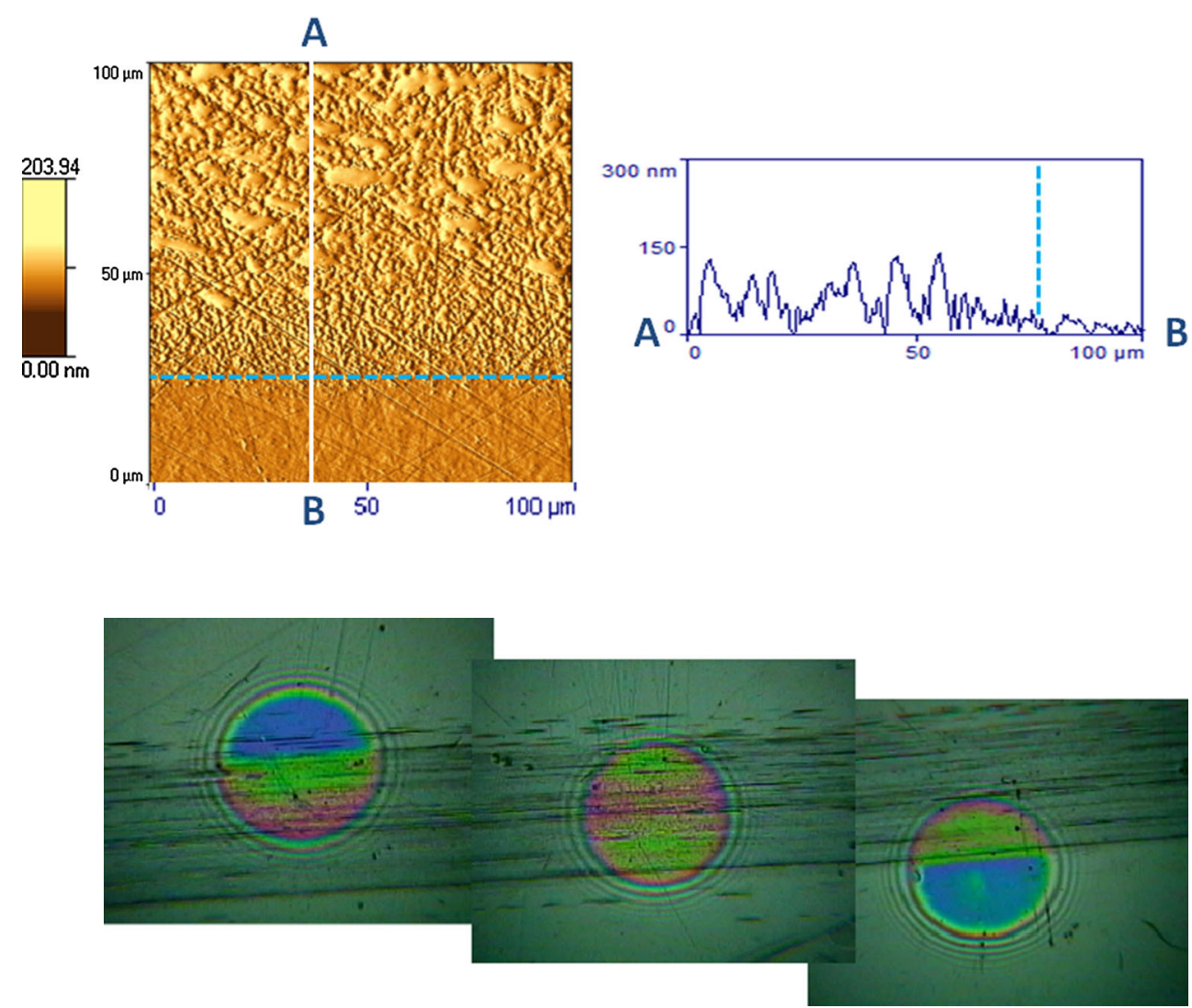

Fig. 3 Optical interference images of ZDDP tribofilm on rubbed track. Adapted from Ref. [9] 
induce cross-linking in a phosphate network leading to a highly connected network with improved mechanical properties. However, the pressures required were far in excess of the yield pressures of most materials and thus unlikely to occur in rubbing contacts. High-pressure infrared studies have also shown no evidence of structural changes in ZDDP up to $21 \mathrm{GPa}$ [22].

It is well known that when solid surfaces rub together, the resulting plastic deformation and fracture processes that occur at contacting asperities generate localised charged regions that result in the emission of energetic particles including photons, electrons, ions and even X-rays [23-25]. When a ZDDP-containing lubricant is present, it has been suggested that this "triboemission" is responsible for the reaction of ZDDP to form tribofilms [26]. While triboemission is quite easy to detect in dry rubbing contact, it has not so far proved possible to observe it in lubricated systems, where any emitted particles are presumed to be captured immediately by the lubricant. This makes it difficult to confirm or refute triboemission's possible impact on tribofilm formation.

\section{Stress-Promoted Thermal Activation}

Quite recently, a few researchers have suggested that the reaction of ZDDP and other additives to form tribofilms may be driven directly by the shear stress present during rubbing. The concept of physical and chemical reactions being driven by applied stress originated in the last century with the work of Prandtl who developed a stress-promoted thermal activation model of crystal plasticity [27]. In the following years, a very similar principle was applied to develop a model of liquid viscosity by Eyring [28], to describe rubber friction by Shallamach [29], to predict the strength of solids by Zhurkov [30] and, in 1978, to model biochemical reactions by Bell [31]. The work of Zhurkov and Bell in particular has led to the development of the modern field of mechanochemistry, specifically devoted to study of the influence of applied force or shear stress on chemical reactions [32, 33]. Since shear stresses are always present in rubbing contacts, the stress-promoted thermal activation concept has a particular relevance to tribology and its history and application have recently been reviewed by one of the authors [34].

In the absence of an applied stress, the influence of temperature on the probability of molecules undergoing many physical and chemical processes (bond breaking, diffusion etc.) is governed by the Boltzmann distribution to give an Arrhenius expression of the form;

Probability $=A e^{-E_{o} / k_{\mathrm{B}} T}$ where $E_{o}$ is the activation energy for the process, $k_{\mathrm{B}}$ the Boltzmann constant, $T$ the absolute temperature, and $A$ is a pre-factor.

According to the stress-promoted thermal activation model, an applied atomic-scale force, $f$, reduces the effective activation energy of a process in the forward direction to give the revised probability equation;

Probability $=A e^{-\left(E_{o}-f \Delta x\right) / k_{\mathrm{B}} T}$

where $\Delta x$ is the activation length. This length has magnitude equivalent to the distance involved in moving a particle or stretching a bond during the atomic-scale process of interest, so $f \Delta x$ represents the mechanical work that augments the relevant thermal energy of an atom or group of atoms.

This effective reduction of activation energy is shown schematically in Fig. 4a, b. Figure $4 \mathrm{a}$ is from Glasstone et al. [35] and illustrates the influence of an applied shear force on forced diffusion of a liquid molecule from a stable position into a neighbouring hole. Upon application of shear force $f$, the energy barrier in the forward direction is reduced by $f \Delta x$ where $\Delta x$ is the distance from the molecule's initial stable position to the top of the activation barrier, the "transition state". The activation energy barrier in the reverse direction is increased.

Figure $4 \mathrm{~b}$ shows a recent representation of the influence of applied force on activation energy in the context of mechanochemistry. It illustrates the impact of applied force, $F_{o}$, on the energy profile along the reaction coordinate, $\xi$, which represents the path the system follows over the potential energy plane from reactant to product. The solid line shows the reaction profile at zero force where $R$ is the reactant, TS is the transition state, and $P$ is the product configuration. The dashed line shows how this is reduced by a factor proportional to the distance over which the force is applied [36].

Equation 2 can also be written in terms of the applied shear stress, $\tau$, to give

Probability $=A e^{-\left(E_{o}-\tau \Delta v\right) / k_{\mathrm{B}} T}$

where $\Delta v$ is now the "activation volume".

These individual particle probability equations can, of course, also be scaled up to molar quantities to give an expression for the rate constant;

Rate constant $=A e^{-(E-N f \Delta x) / R T}$

Equation 4 thus predicts that the rate of the process is increased due to applied force, $f$ by a factor $e^{N f \Delta x / R T}$ or applied shear stress, $\tau$ by $e^{N \tau \Delta v / R T}$. It should be noted that the above equations represent the simplest form of the stress-promoted thermal activation model and several refinements exist, particularly in the context of 


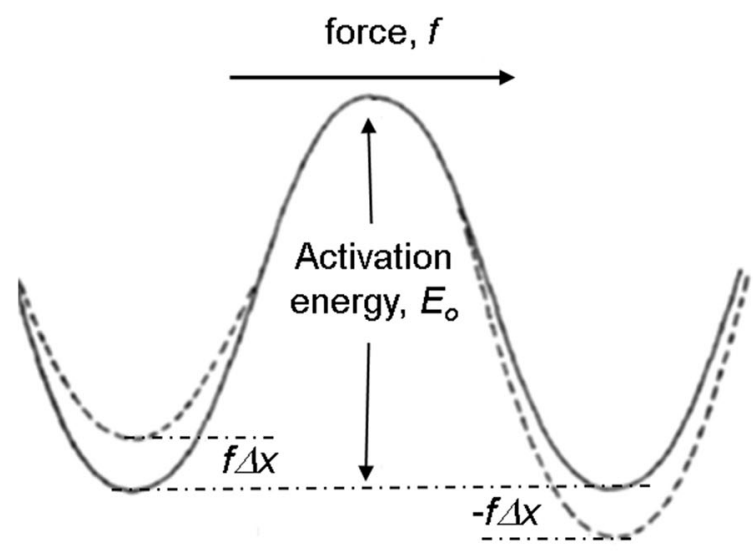

(a)

Fig. 4 a Schematic diagram of influence of applied force on activation energy in forced diffusion of liquid molecules. Adapted from Ref. [35]. b Schematic diagram of influence of applied force on

mechanochemistry, that take account, for example, of the shape of the energy barrier and the influence of applied force upon this [36, 37].

Gosvami et al. [17] studied the influence of normal force and temperature on ZDDP film formation on silicon using an atomic-force microscope (AFM) and showed that the kinetics of film formation were consistent with a stresspromoted thermal activation model, while Ghanbarzadeh et al. [38] employed an expression equivalent to Eq. 4 in a simulation of ZDDP film formation and removal and thus wear. Felts et al. [39] used an AFM to show that the influence of applied shear stress and rubbing time on the removal of bonded oxygen atoms from graphene and consequent reduction in friction was consistent with the stress-promoted thermal activation model. Recently, Adams et al. [40] have also shown the rate of decomposition of adsorbed methyl thiolate on copper during rubbing, as measured by the rate of methane emission, is consistent with this model.

As will be discussed later in this paper, the application of this model to explain ZDDP tribofilm formation is appealing since it is able to explain several important features of ZDDP films. Unfortunately, there is a considerable practical problem in proving conclusively that ZDDP film formation is in fact controlled directly by stress-promoted thermal activation since tribofilms generally form in rubbing contacts where all the other possible influencing factors occur in parallel. For example, triboemission is known to result from plastic deformation of contacting asperities, and plastic deformation itself has been modelled using a stress-promoted thermal activation model. A conclusive proof that ZDDP film formation is promoted directly by shear stress and is thus a manifestation of mechanochemistry requires

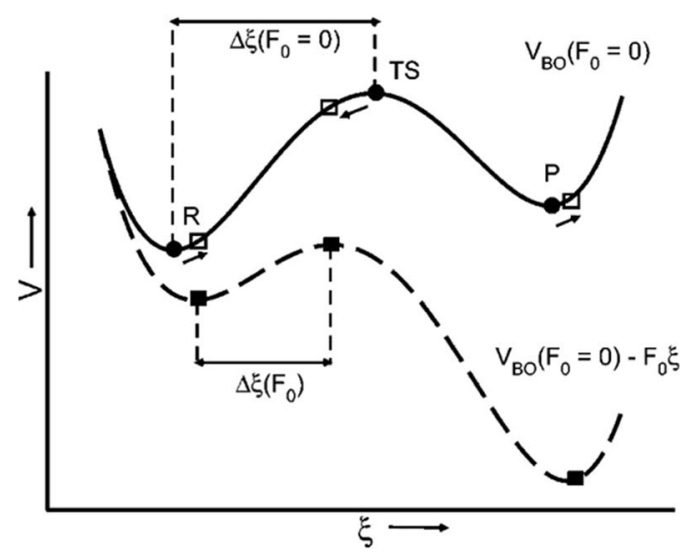

(b)

activation energy in a chemical reaction [36]. Dashed lines are the energy barrier shapes resulting from the application of force

this film formation to take place in the absence of all other possible drivers or that the rate at which the ZDDP film is formed varies with shear stress when all other possible drivers are held constant. This paper describes an experimental study aimed at showing that ZDDP is indeed controlled by shear stress in accord with the stress-promoted thermal activation model.

\section{Experimental Approach}

In thin-film rubbing contacts, ZDDP film formation is generally assumed to begin at sliding asperity contacts and it has been shown that in steel/steel rolling contacts at modest pressures film formation does not normally occur if the surfaces are separated by a liquid lubricating film sufficient to give the lambda ratio (ratio of hydrodynamic film thickness to composite surface roughness) significantly greater than unity [6]. If, however, ZDDP film formation were driven solely by applied shear stress, then in principle it would occur even in full film lubrication conditions if the fluid shear stress were high enough. If it can be shown that ZDDP film formation does in fact occur when the rubbing surfaces are fully separated by a fluid film, this precludes the possibility of asperity plastic deformation and thus triboemission driving the ZDDP reaction. Furthermore, if it can be shown to occur in low-sliding-speed contacts in full film conditions, this eliminates any possible effect on ZDDP film formation of flash temperature. Finally, if it can be shown to occur only for fluids that give high shear stress and not for ones that have low shear stress at the same pressure, this will indicate that shear stress rather than applied pressure drives the ZDDP film formation. 
The experimental approach adopted in this study is thus to test and compare the ability of ZDDP solutions to form tribofilms in elastohydrodynamic (EHD) lubricated conditions in a rolling-sliding, ball-on-disc rig at high entrainment speed (to ensure high EHD film thickness), low sliding speed (to ensure negligible flash temperature) using both high- and low-EHD friction base fluid solvents.

\section{Test Methods}

Rolling-sliding EHD tests were carried out using a minitraction machine-spacer layer imaging set-up (MTM2SLIM, PCS Instruments, Acton, UK). In this, a ball is loaded and rotated against the flat surface of a rotating disc immersed in lubricant at a controlled temperature as shown schematically in Fig. 5. Ball and disc are driven by independent motors to enable any combination of sliding and rolling. Thus, it is possible to combine a high entrainment speed, and so a thick EHD film, with a small speed difference between the two surfaces, i.e. a low sliding speed. The drive motors, applied load and temperature are all computer-controlled, and friction is measured from a load cell attached to the ball drive shaft.

The SLIM attachment enables the formation of any tribofilm on the ball to be monitored throughout a test by periodically halting rotation, unloading the ball from the disc and uploading it against a glass window coated with a semi-reflecting film and a spacer layer to capture an optical interference image of the ball track.

In each test, the ball and disc were rubbed together at a fixed entrainment speed, slide-roll ratio (ratio of sliding speed to entrainment speed), applied load and temperature for $4 \mathrm{~h}$ while periodically using SLIM to monitor the formation of a tribofilm on the ball. At the end of each test, the film was further explored ex situ using an atomic-force microscope (AFM) and SEM EDX surface analysis.

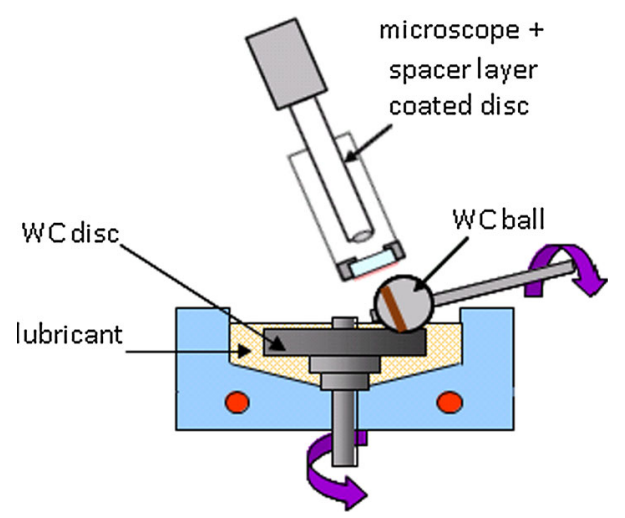

Fig. 5 MTM-SLIM set-up. The rubbed track on the ball is shown in brown (Color figure online)
The main test conditions are listed in Table 1 . The choices of entrainment speed and slide-roll ratio are explained further below. It was recognised that in order to achieve shear stresses comparable to those likely to be present at asperity contacts, high pressure was essential. Most ZDDP tribofilm formation studies, as shown for example in Figs. 2 and 3, have been carried out with a steel ball on a steel disc using an applied load to produce a mean Hertz pressure of typically $1 \mathrm{GPa}$. However, as can be seen in Fig. 2, the film formed by ZDDP is very rough so that the actual area of contact in thin-film conditions will be lower than the apparent area of contact. Thus, the local asperity pressure will be considerably higher than $1 \mathrm{GPa}$, and, based on estimates of pad area, lie in the range 2-4 GPa. In their AFM study formation, Gosvami et al. [17] observed ZDDP film formation over the normal force range 2.5-5 GPa. Unfortunately, the MTM2 has a maximum load of $75 \mathrm{~N}$ and at this load the maximum Hertz pressure in steel/steel contacts cannot exceed $1.2 \mathrm{GPa}$ using a ball-on-flat contact with the smallest ball that can be accommodated. Higher loads are possible if a barrelled specimen is used, but this forms an elliptical contact elongated in the entrainment direction. Such a configuration results in a very thin EHD film, so it is not possible to ensure full EHD film conditions. In order to approach the pressures likely to be present at asperity contacts in a ballon-flat contact, tests were therefore carried out using tungsten carbide (WC) balls and discs whose high elastic modulus gives a significantly higher contact pressure than steel at the same applied load.

The WC balls were $19.0 \mathrm{~mm}$ diameter, and the balls and discs had an elastic modulus of $630 \mathrm{GPa}$ and Poisson's ratio 0.21 . Root mean square ball roughness, $R_{q}$, was $10.0 \mathrm{~nm}$ and disc roughness $7.0 \mathrm{~nm}$, giving a composite surface roughness of $12.2 \mathrm{~nm}$. Two tests were carried out on each ball and disc by reversing the ball in its holder and using both sides of the disc. Ball and disc were cleaned just prior to use in an ultrasonic bath using toluene and then Analar acetone.

Table 2 lists the loads used in this study and the corresponding mean and maximum pressures and contact radii calculated from Hertz theory [41]. The pressures lie at the low end of the range over which other work suggests that ZDDP films are likely to form.

Table 1 Conditions for full EHD ZDDP film formation tests

\begin{tabular}{ll}
\hline Entrainment speed & $3.0,3.1 \mathrm{~m} / \mathrm{s}$ \\
Slide-roll ratio & $3.0 \%$ \\
Sliding speed & $0.09 \mathrm{~m} / \mathrm{s}$ \\
Temperature & $60-120{ }^{\circ} \mathrm{C}$ \\
Applied load & $20-75 \mathrm{~N}$ \\
\hline
\end{tabular}


Table 2 Contact loads used and corresponding Hertz pressures and radii for $19 \mathrm{~mm}$ diameter WC ball-on-flat contact

\begin{tabular}{llll}
\hline Applied load $N$ & Max. Hertz pressure (GPa) & Mean Hertz pressure $(\mathrm{GPa})$ & Hertz contact radius $(\mu \mathrm{m})$ \\
\hline 20 & 1.65 & 1.1 & 76 \\
50 & 2.25 & 1.5 & 103 \\
55 & 2.35 & 1.55 & 106 \\
60 & 2.4 & 1.6 & 109 \\
75 & 2.6 & 1.7 & 118 \\
\hline
\end{tabular}

In order to ensure full film EHD conditions during MTM tests, EHD film thickness was measured using ultrathinfilm interferometry (PCS Instruments) for both of the test lubricants over the entrainment speed and temperature conditions of interest. These were carried out in nominally pure rolling using a WC ball on sapphire disc at a mean Hertz pressure of $1.12 \mathrm{GPa}$.

\section{Test Fluids}

Two hydrocarbon base fluids were employed: one designed to give high EHD friction and other low EHD friction. DM2H (2,3-dimethyl-2-[(3-methylbicyclo[2.2.1]hept-2yl)methyl]bicyclo[2.2.1]heptane) is an additive-free, hightraction base fluid supplied by Idemitsu Kosan with the molecular structure shown in Fig. 6a [42]. The viscosity of DM2H was $22.7 \mathrm{cSt}$ at $40{ }^{\circ} \mathrm{C}$ and $4.07 \mathrm{cSt}$ at $100^{\circ} \mathrm{C}$. At $120^{\circ} \mathrm{C}$, its dynamic viscosity was $2.56 \mathrm{mPas}$.

The low EHD friction base oil was a polyalphaolefin (PAO) obtained by mixing two PAOs of viscosity $5.9 \mathrm{cSt}$ and $7.1 \mathrm{cSt}$ at $100{ }^{\circ} \mathrm{C}$ to form a blend having same EHD film thickness as the $\mathrm{DM} 2 \mathrm{H}$ at the maximum temperature studied of $120^{\circ} \mathrm{C}$. The viscosity of the resulting PAO blend was $33.6 \mathrm{cSt}$ at $40{ }^{\circ} \mathrm{C}, 6.38 \mathrm{cSt}$ at $100{ }^{\circ} \mathrm{C}$ and 4.49 $\mathrm{mPas}$ at $120^{\circ} \mathrm{C}$. A PAO blend of higher viscosity than DM2H was needed to give the same EHD film thickness since the former has a lower pressure-viscosity coefficient than the latter. The PAOs used were both hydrogenated

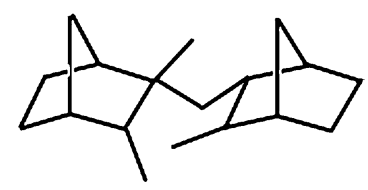

(a)

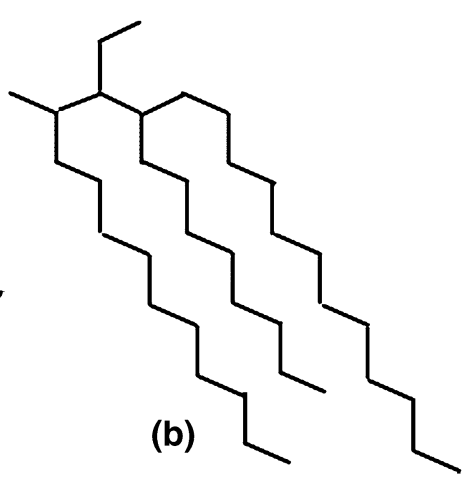

Fig. 6 a Molecular structure of DM2H, b representative molecular structure of PAO oligomers of n-dodec-1-ene molecules, and a typical molecular structure of a trimer is shown in Fig. 6b. Due to their predominantly linear hydrocarbon chain structure, they give relatively low EHD friction.

One commercial mixed ZDDP was studied containing $30 \%$ primary and $70 \%$ secondary alkyl groups. In all tests, it was used at a concentration of $800 \mathrm{ppm}$ phosphorus.

\section{Preliminary Tests}

A preliminary test was carried out to show that ZDDP is able to form films on WC in a similar fashion to steel when the surfaces are rubbed together under thin-film conditions. WC ball and disc were immersed in ZDDP solution in PAO and rubbed together at a low entrainment speed of $0.05 \mathrm{~m} /$ s, $50 \%$ slide-roll ratio, $31 \mathrm{~N}$ load and $120{ }^{\circ} \mathrm{C}$. Based on film thickness measurements, the theoretical initial lambda ratio at this entrainment speed was 0.25 , indicative of mixed lubrication conditions. Periodically, ball and disc were halted and a SLIM image taken from the ball. Figure 7 shows a series of such SLIM images. A film forms very rapidly on the rubbing track and grows to reach a mean thickness of ca $60 \mathrm{~nm}$ after $45 \mathrm{~min}$. After this time, localised patches of the film are lost. This latter behaviour is not seen with most ZDDPs in steel/steel contacts and may result from a combination of weak adhesion of the film with the much higher than normal pressure in the WC/ WC contacts compared to the steel/steel ones generally tested. This behaviour was not seen in the thick EHD film work described later in this paper.

EHD film thickness measurements were carried out on the two base fluids at $120{ }^{\circ} \mathrm{C}$. Results are shown in Fig. 8 . It can be seen that both lubricants form almost identical EHD film thicknesses although those of DM2H are very slightly lower than PAO.

Based on these measurements, subsequent ZDDP solution testing was carried out at an entrainment speed of $3.0 \mathrm{~m} / \mathrm{s}$ for the PAO solution and $3.1 \mathrm{~m} / \mathrm{s}$ for the DM2H. The speed difference was to ensure identical EHD film thicknesses for the two solutions. Optical interferometry indicates a film thickness of $68 \mathrm{~nm}$ at $120{ }^{\circ} \mathrm{C}$ for both fluids at $30 \mathrm{~N}$ in a $\mathrm{WC} /$ sapphire contact. Based on EHD 


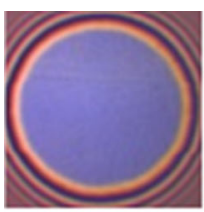

$0 \mathrm{~min}$.

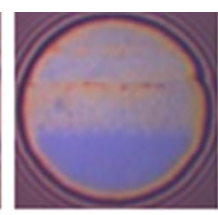

5 min.

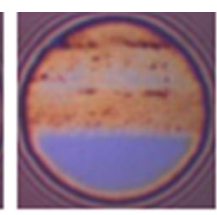

$10 \mathrm{~min}$.

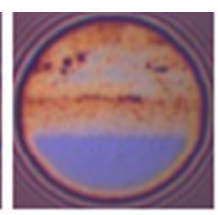

$15 \mathrm{~min}$.

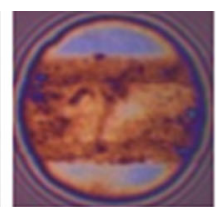

$30 \mathrm{~min}$.

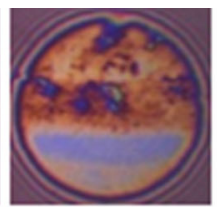

$45 \mathrm{~min}$.

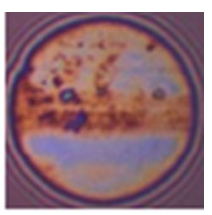

$60 \mathrm{~min}$.

Fig. 7 SLIM images showing ZDDP tribofilm formation in a thin-film rolling/sliding WC/WC contact at $120{ }^{\circ} \mathrm{C}$

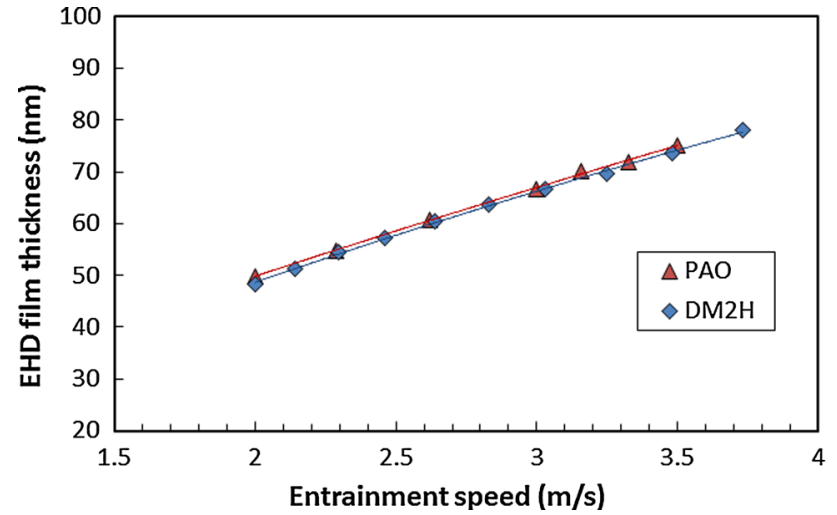

Fig. 8 EHD film thickness of PAO and DM2H at $120{ }^{\circ} \mathrm{C}$ and $30 \mathrm{~N}$ load in a rolling WC ball on sapphire disc contact at $120{ }^{\circ} \mathrm{C}$

film thickness depending on load to the power -0.073 and on reduced elastic modulus to the power -0.12 , in accord with EHD theory [43], this corresponds to a film thickness of $64 \mathrm{~nm}$ at $50 \mathrm{~N}$ load and $62 \mathrm{~nm}$ at $75 \mathrm{~N}$ load in a WC/ WC contact. Since the composite surface roughness of the WC ball and disc was $12.2 \mathrm{~nm}$, this implies a minimum lambda ratio (ratio of EHD film thickness to surface roughness) of 5.1; i.e. full EHD film conditions.

Preliminary tests were also carried out to measure the EHD friction properties of the two base fluids. For these, the entrainment speed was held constant at 3.0 or $3.1 \mathrm{~m} / \mathrm{s}$ and friction coefficient measured while progressively increasing the slide-roll ratio from zero to $20 \%$ to obtain "traction curves". Tests were carried out at several temperatures and applied loads. Figure 9a compares friction coefficient versus slide-roll ratio curves of the two fluids at $120{ }^{\circ} \mathrm{C}$ and three applied loads, while Fig. 9b compares them at $75 \mathrm{~N}$ load and three temperatures. For PAO, friction coefficient increases with slide-roll ratio and applied load and decreases with temperature. This type of behaviour is typical of most base fluids in elastohydrodynamic conditions. It is evident that the DM2H gives a much higher friction coefficient than PAO at all conditions and also that, compared to PAO, its friction coefficient is much less sensitive to load and temperature over the range studied. The fact that the friction coefficient is independent of load for EHD films of DM2H indicates that the shear stress is proportional to the load for this fluid.
For the purposes of this study, the property of primary interest was not the friction coefficient, but the shear stress. The mean shear stress is simply the measured friction divided by the contact area where the latter is, for a ball-onflat the contact, a circle of area $\pi a^{2}$ where $a$ is the Hertz contact radius.

Figures 10a, b show how mean shear stress varies with slide-roll ratio for the two fluids at the three test temperatures and loads. The mean shear stress clearly depends strongly on load, but at all loads the shear stress with PAO is much less than that with DM2H. It should be noted that, based on Hertz theory when shear stress is proportional to load as it is for DM2H, the maximum shear stress, which in the absence of significant shear heating occurs where the pressure is highest close to the centre of the contact, will be 1.5 times higher than the mean shear stress.

Based on these results, a slide-roll ratio of $3 \%$ was selected for subsequent ZDDP film formation work. This is high enough for DM2H to be close to the maximum of its EHD friction curve and thus provides a large difference in shear stress between the two fluids, but is low enough to avoid significant frictional heating. Based on flash temperature theory [44], the mean oil film temperature rise in the contact was calculated to be $3.3{ }^{\circ} \mathrm{C}$ for $\mathrm{DM} 2 \mathrm{H}$ and $0.7{ }^{\circ} \mathrm{C}$ for PAO at $75 \mathrm{~N}$ and $120{ }^{\circ} \mathrm{C}$.

\section{ZDDP Film Formation Results}

Figure 11 compares MTM SLIM images from high entrainment speed, thick film EHD tests carried out at $75 \mathrm{~N}$ and $120{ }^{\circ} \mathrm{C}$ for ZDDP solution in the two base fluids. The mean shear stresses, $\tau_{m n}$, are shown, calculated from the product of measured EHD friction coefficient and mean pressure. At these high pressures, the contact dimensions as well as the pressures in full EHD film conditions can be assumed to be almost identical to the Hertz solution. No detectible tribofilm was measured for ZDDP in PAO while a ZDDP film developed in the central region of the contact for ZDDP in DM2H. It should be noted that the EHD film thickness and Hertz contact pressure were the same for both tests; the only difference was the nature of the base fluid. Repeat tests with fresh solutions and specimens showed similar behaviour. 


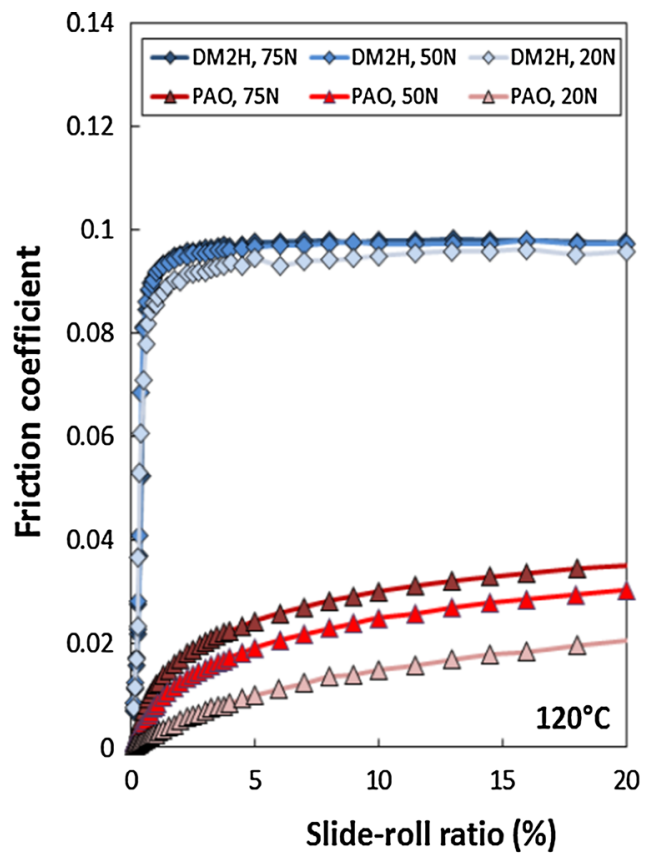

(a)

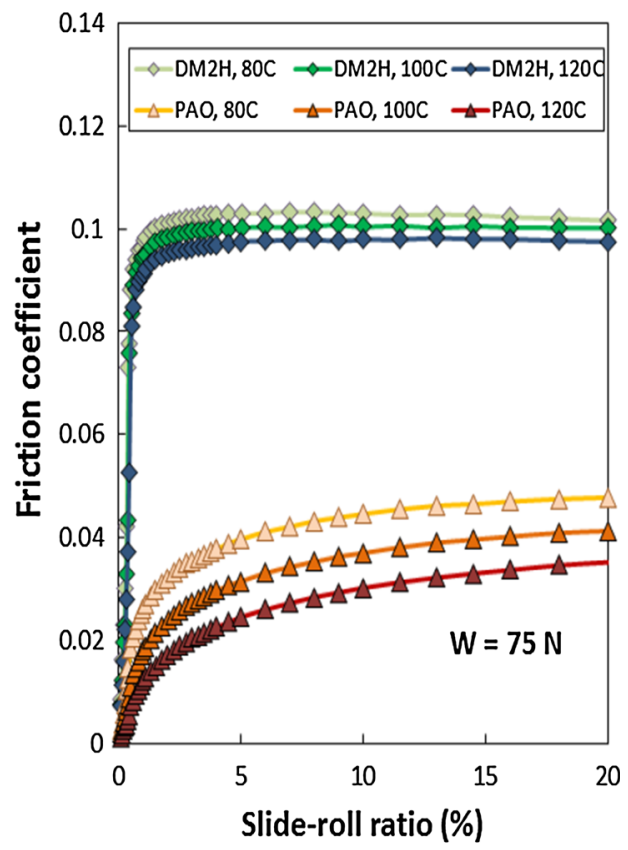

(b)

Fig. 9 EHD friction coefficient versus slide-roll ratio for the two base fluids at $\mathbf{a}$ fixed load and three temperatures and $\mathbf{b}$ fixed temperature and three loads

Fig. 10 Mean shear stress versus slide-roll ratio for the two base fluids at a fixed load and three temperatures and b fixed temperature and three loads

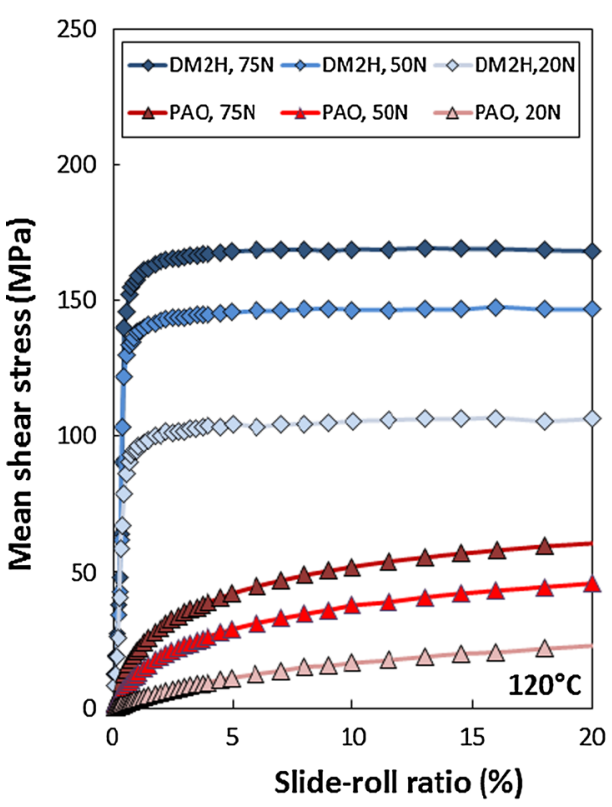

(a)

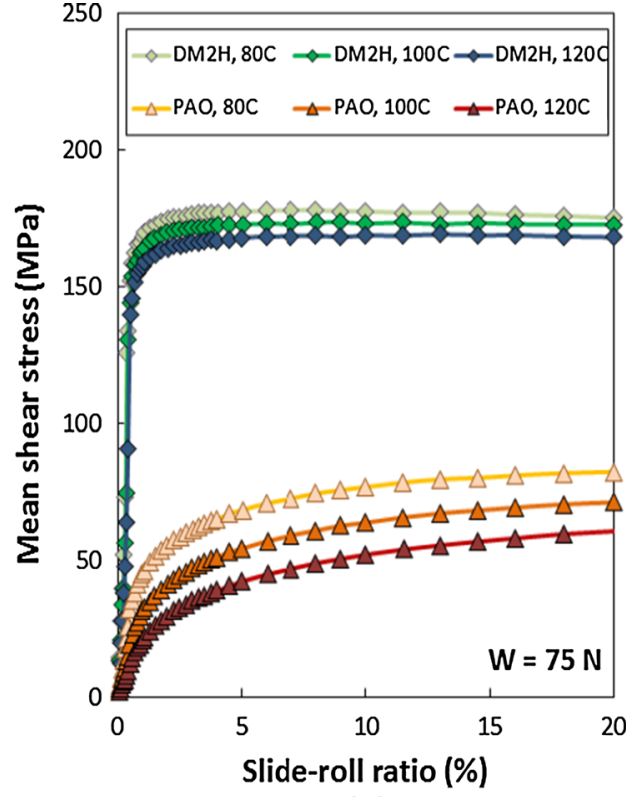

(b)
Figure 12 compares tests on the two solutions at the same load, but a lower temperature of $100{ }^{\circ} \mathrm{C}$. As might be expected, ZDDP solution in PAO does not form a film. However, ZDDP in DM2H does form a tribofilm, albeit more slowly than at $120^{\circ} \mathrm{C}$.

This indicates that the difference between ZDDP behaviour in PAO and DM2H seen in Fig. 11 does not result simply from the slightly greater temperature rise of the DM2H solution within the contact.

Figure 13 compares ZDDP film formation from DM2H at five applied loads. A film forms at 75, 60, 55 and $50 \mathrm{~N}$, but negligible film forms within $4 \mathrm{~h}$ rubbing at $20 \mathrm{~N}$ load.

The variation of mean ZDDP film thickness with rubbing time was calculated from the images in Figs. 12 and 
PAO $75 \mathrm{~N}$,

$\left(\tau_{m n}=35 \mathrm{MPa}\right)$

$120^{\circ} \mathrm{C}$

DM2H $75 \mathrm{~N}$,

$\left(\varepsilon_{\mathrm{mn}}=165 \mathrm{MPa}\right)$ $120^{\circ} \mathrm{C}$

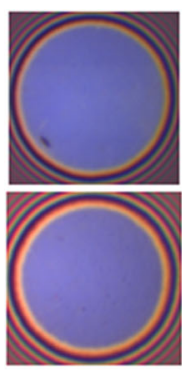

0 min.

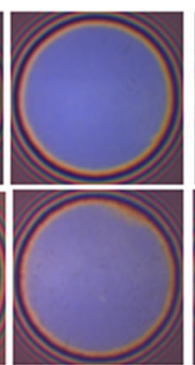

5 min.

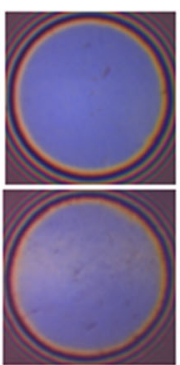

$15 \mathrm{~min}$.

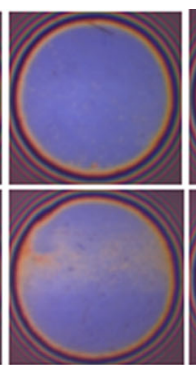

$30 \mathrm{~min}$.

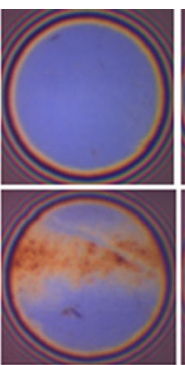

$60 \mathrm{~min}$.

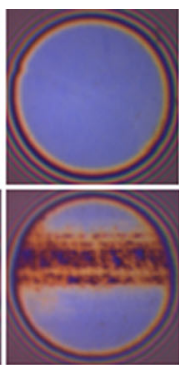

$120 \mathrm{~min}$.

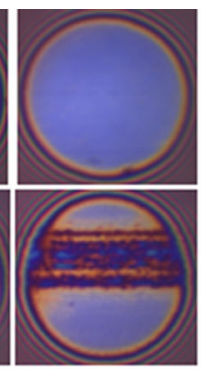

$180 \mathrm{~min}$.

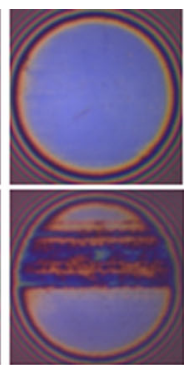

$240 \mathrm{~min}$.

Fig. 11 Comparison of SLIM images for ZDDP solution in PAO and DM2H over a $4 \mathrm{~h}$ test at $75 \mathrm{~N}$ and $120{ }^{\circ} \mathrm{C} .(U=3 \mathrm{~m} / \mathrm{s}$, SRR $=3 \%)$

\section{PAO $75 \mathrm{~N}$, $\left(\tau_{m n}=35 \mathrm{MPa}\right)$ $100^{\circ} \mathrm{C}$}

$\mathrm{DM} 2 \mathrm{H} 75 \mathrm{~N}$, $\left(\tau_{m n}=165 \mathrm{MPa}\right)$ $100^{\circ} \mathrm{C}$
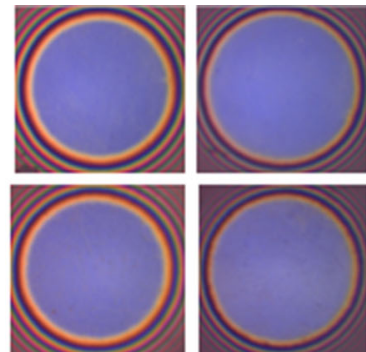
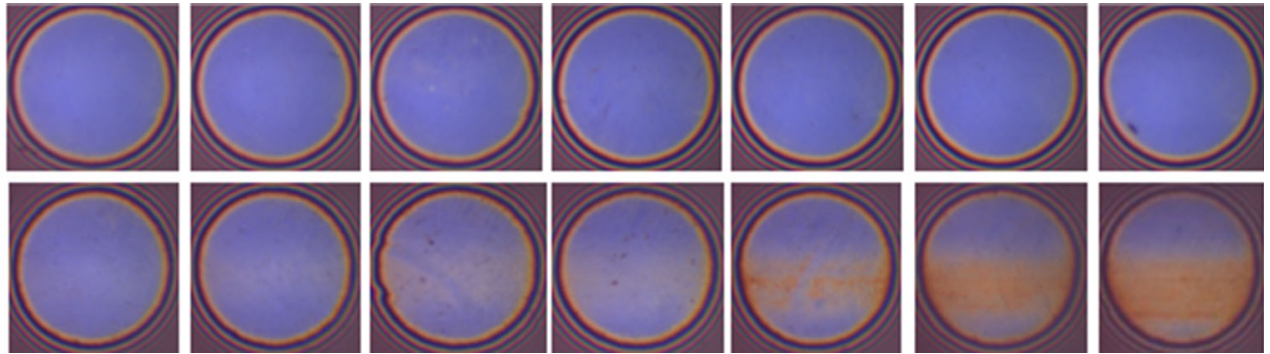

Fig. 12 Comparison of SLIM images for ZDDP solution in PAO and DM2H over a $4 \mathrm{~h}$ test at $75 \mathrm{~N}$ and $100{ }^{\circ} \mathrm{C} .(U=3 \mathrm{~m} / \mathrm{s}$, SRR $=3 \%)$

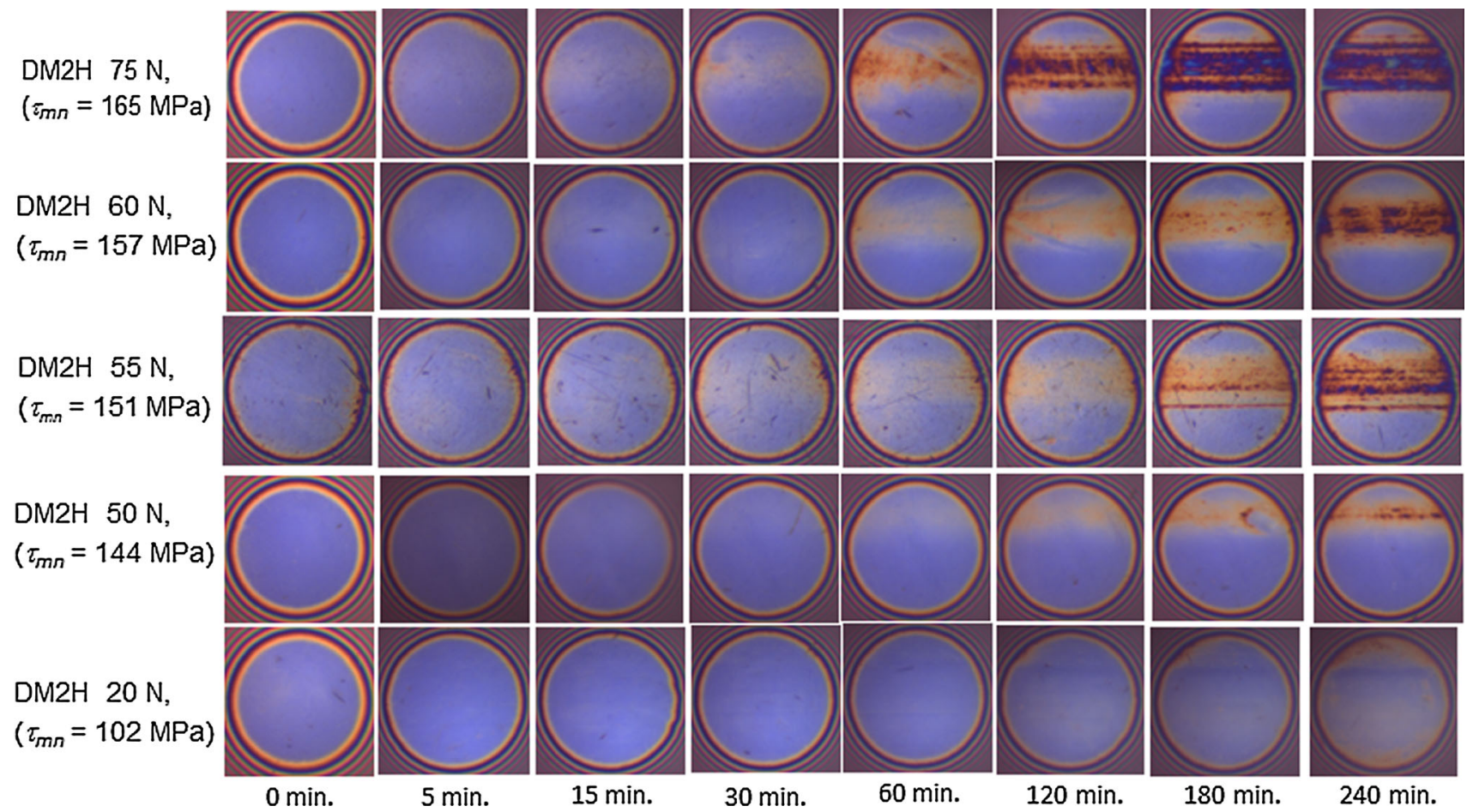

Fig. 13 Comparison of SLIM images for ZDDP solution in DM2H over a $4 \mathrm{~h}$ test at $120{ }^{\circ} \mathrm{C}$ and four applied loads. $(U=3 \mathrm{~m} / \mathrm{s}$, SRR $=3 \%)$

13. These thicknesses were calculated only from the tribofilm region itself and are shown in Fig. 14. The error bars show $15 \%$ variation which was found during three repeat tests at $75 \mathrm{~N}$ and $120^{\circ} \mathrm{C}$. It can be seen that film thickness increases approximately linearly with rubbing time except when the films become locally very thick, 


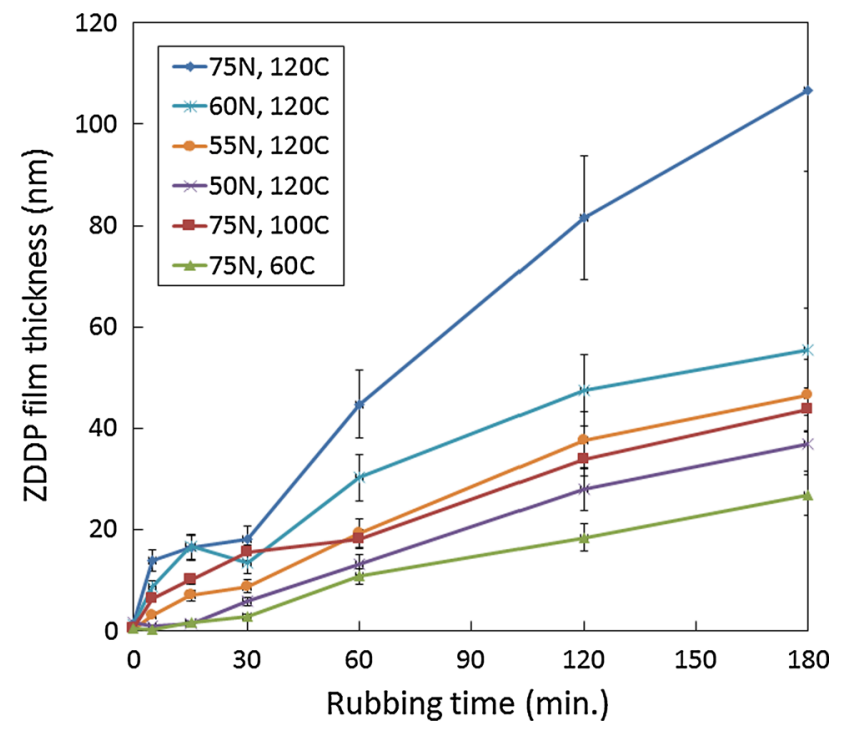

Fig. 14 Variation of mean tribofilm thickness (within the film buildup region) with rubbing time for solution of ZDDP in DM2H

which occurred at after extended rubbing at high temperature.

Figure 15 shows AFM contact mode images from the central region of the tribofilm track after $4 \mathrm{~h}$ tests at $120{ }^{\circ} \mathrm{C}$ and three applied loads. For thin films, the initial ZDDP film formed is quite smooth (in Fig. 15a root mean square roughness $R_{q}=5.5 \mathrm{~nm}$ and in Fig. $15 \mathrm{~b}, R_{q}=10.5 \mathrm{~nm}$ ) and comparable to the initial ball roughness, though of shorter wavelength. When a thick film develops, as in Fig. $15 \mathrm{c}$, it is probable that the pressure and thus the shear stress distribution become considerably affected by the film topography and the contact moves towards mixed lubrication conditions.

SEM EDX was used to analyse the films formed at the end of the $4 \mathrm{~h}$ rubbing tests at $75 \mathrm{~N}$ and $120{ }^{\circ} \mathrm{C}$. This showed the expected presence of $\mathrm{P}, \mathrm{S}$ and $\mathrm{Zn}$, with a preponderance of $\mathrm{P}$.

From the above, it is clear that ZDDP forms tribofilms much more easily from the high-EHD-friction fluid DM2H than from the low-friction one PAO. To confirm that this was not due simply to an enhanced chemical reactivity of the ZDDP in the DM2H, the formation of thermal films by ZDDP in the two fluids was studied. Tungsten carbide balls were immersed in ZDDP solutions at $150{ }^{\circ} \mathrm{C}$ for $10 \mathrm{~h}$. Periodically, the balls were withdrawn and mounted in an MTM SLIM to measure any reaction film. The measured mean film thickness results are shown in Fig. 16, along with the interference images after 5 and $10 \mathrm{~h}$. Thermal film thickness develops linearly over time to reach $32 \mathrm{~nm}$ for ZDDP in DM2H after $10 \mathrm{~h}$ and $135 \mathrm{~nm}$ for ZDDP in PAO. In terms of thermal film formation at high temperature, it is clear that ZDDP is significantly more reactive in PAO than DM2H, possibly because of PAO's lower polarity.

The AFM topography image of the thermal film formed after $10 \mathrm{~h}$ from ZDDP solution in DM2H had similar morphology to that formed in thick EHD film conditions at $60{ }^{\circ} \mathrm{C}$ (Fig. 4a), with a lower roughness of $R_{q}=3.1 \mathrm{~nm}$.

\section{Discussion}

These tests support the hypothesis that ZDDP tribofilm formation in rubbing contact is driven by applied shear stress and is thus a manifestation of mechanochemistry. The finding that a ZDDP tribofilm is formed in thick film conditions when no solid-solid contact occurs suggests that triboemission does not drive ZDDP film formation in this study. The fact that a tribofilm is formed from ZDDP in a high-EHD-friction fluid, but not from ZDDP in a low one at otherwise identical conditions suggests that shear stress rather than pressure is the driving factor.

It is noteworthy that the tribofilm tracks formed in thick EHD film conditions as shown in Fig. 13 are narrower than those formed in mixed lubrication conditions as shown in Fig. 7. The overall diameter of the WC/glass interference images is $257 \mu \mathrm{m}$, and from this the widths of the film tracks can be estimated and compared to the corresponding WC/WC Hertzian contact diameters. For the tribofilm formed in mixed lubrication conditions, as shown for example in the 30 min rubbing image in Fig. 7, the track width was equal to the Hertzian contact diameter at the test
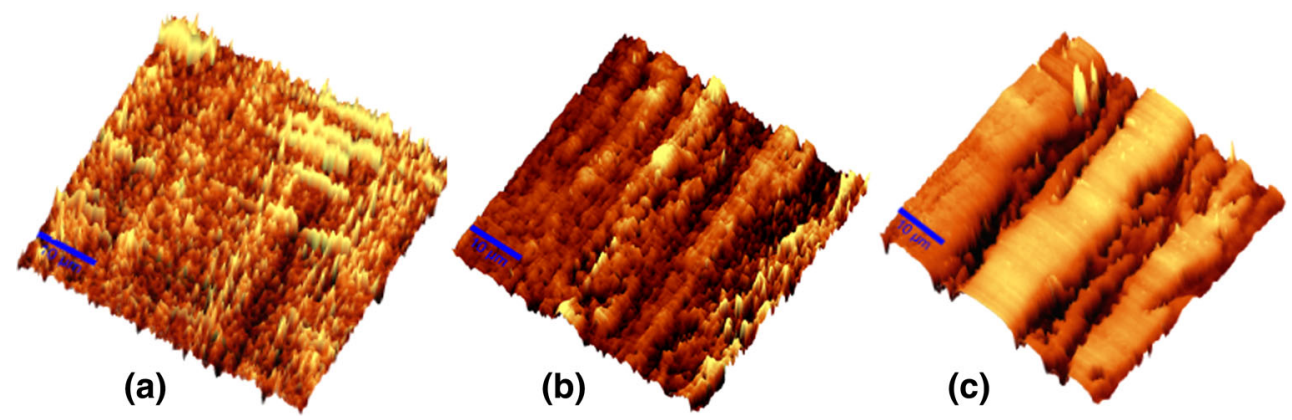

Fig. 15 AFM images of tribofilm after 4 h rubbing in thick EHD film conditions at $75 \mathrm{~N}$. a $60{ }^{\circ} \mathrm{C}, \mathbf{b} 100{ }^{\circ} \mathrm{C}$, c $120{ }^{\circ} \mathrm{C}$. Blue lines are $10 \mu \mathrm{m}$ (Color figure online) 


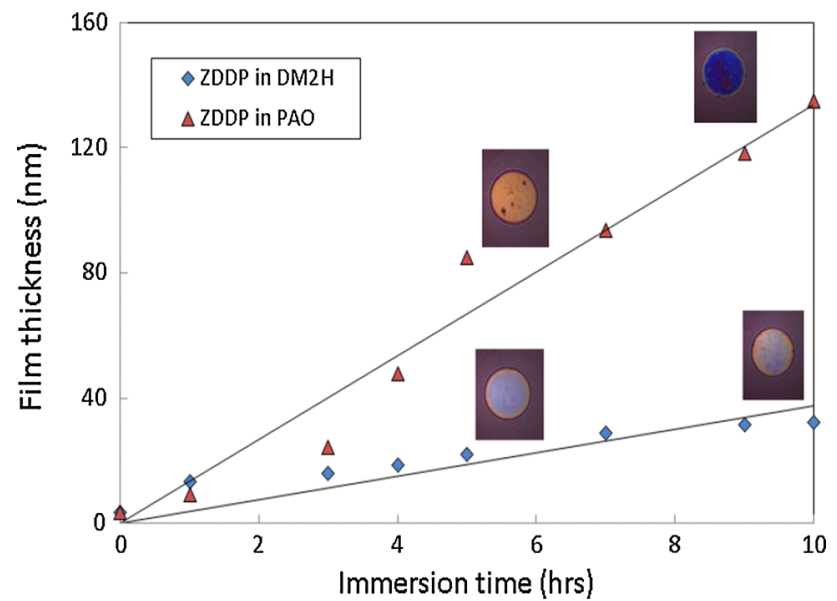

Fig. 16 Growth of thermal films from ZDDP solutions at $150^{\circ} \mathrm{C}$. Images shown are after 5 and $10 \mathrm{~h}$ immersion

load of $31 \mathrm{~N}(175 \mu \mathrm{m})$ indicating that a film is formed over the whole rubbing contact. However, from the $240 \mathrm{~min}$ images in Fig. 13 obtained in thick EHD film conditions, the track width was about half the Hertzian contact width at $75 \mathrm{~N}$, one-third of this at $60 \mathrm{~N}$ and only 0.19 of the Hertzian diameter at $50 \mathrm{~N}$. Assuming that the shear stress varies in proportion to the Hertz pressure across the contact so that

$\tau=\tau_{m x}\left(1-r^{2} / a^{2}\right)^{0.5}$

it is straightforward to estimate the shear stress at the edge of the tribofilm track. At all three loads, this was ca $140 \mathrm{MPa}$, suggesting that this shear stress corresponds to a threshold value below which the rate of tribofilm formation at $120{ }^{\circ} \mathrm{C}$ is negligible.

If the stress-promoted thermal activation equation

Rate $=A e^{-(E-N \tau \Delta v) / R T}$

is obeyed, then at a fixed temperature the rate of ZDDP film build-up should be related to the shear stress by

$\log ($ rate $)=\log (A)-\frac{E}{R T}+\frac{N \Delta v}{R T} \tau$

Figure 17 shows a plot of $\log$ (tribofilm formation rate) versus maximum shear stress from the tests carried out at $120{ }^{\circ} \mathrm{C}$. The rate of film formation was obtained by fitting a straight line to the plots in Fig. 14, excluding data for film thicknesses above $50 \mathrm{~nm}$ since for such thick films there is a likelihood of some direct solid-solid contact. It is assumed that the ZDDP film volume is proportional to the mean film thickness when the latter is obtained by averaging over the film growth region. The error bars indicate a standard deviation in film formation rate of $7 \%$ which was determined from three repeat tests at $120{ }^{\circ} \mathrm{C}, 75 \mathrm{~N}$ load.

From the gradient of Fig. 17, the activation volume is calculated to be $0.18 \mathrm{~nm}^{3}$. This value is considerably

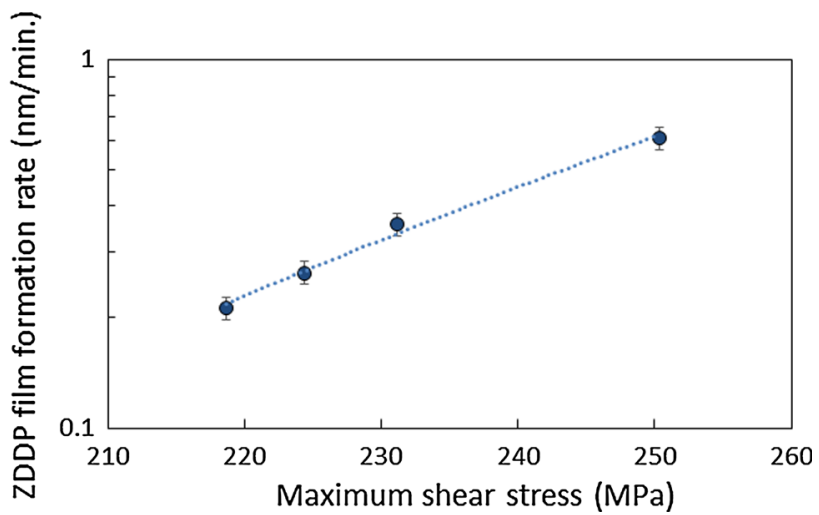

Fig. 17 Plot of log (ZDDP film formation rate) versus maximum shear stress for tests of ZDDP solution in DM2H at $120^{\circ} \mathrm{C}$

higher than that calculated by Gosvami et al. [17] of $0.0038 \mathrm{~nm}^{3}$, but these authors used mean pressure rather than shear stress in their evaluation and also note that their calculated pressures are an upper bound since they do not take account of the influence of the ZDDP film itself on the contact pressure. It should be noted that the activation length, $\Delta x$ in Eq. 4, cannot be determined simply by taking the cube root of the activation volume since, as indicated by Eyring [28], the latter is not a true volume but rather the product of the area of the molecule over which the shear stress acts to impart a force and the activation length in the same plane through which this force acts. If we consider a typical bond length of $0.2 \mathrm{~nm}$, this implies a molecular area of $1 \mathrm{~nm}^{2}$ over which shear stress acts, which is not unrealistic for a dialkyldithiophosphate group. $N \Delta v \tau$ represents the contribution of mechanical force to lowering the molar activation energy. At $75 \mathrm{~N}$, when $\tau_{\max }=250 \mathrm{MPa}$, this corresponds to a value of $27 \mathrm{~kJ} / \mathrm{mole}$. In practical terms, the effect of this at $250 \mathrm{MPa}$ is to increase the rate of ZDDP reaction by $e^{N \Delta v \tau / R T}$, i.e. by a factor of almost 4000 .

It is of interest to compare this effect of shear stress with the thermal activation energy $E$, which can be estimated from the three DM2H tests at the same shear stress and different temperatures by plotting $\log ($ rate $)-N \Delta v \tau / R T$ against $1 / T$. This yields $E=53 \mathrm{~kJ} / \mathrm{mol}$, suggesting that at the highest applied load the applied shear stress effectively decreases the thermal activation energy by half.

A mechanochemical mechanism of ZDDP tribofilm formation is able to explain several important features of ZDDPs. Firstly, of course, it rationalises the relationship between thermal and tribofilms; suggesting that the latter are simply thermal films in which reaction has been promoted by the applied frictional force to take place at lower temperatures. Any differences between the two, which appear to largely concern their mechanical properties, arise only from the different rates and conditions under which they form and possibly a greater proportion of iron cations 
emanating from the substrate due to mechanical action, and consequently a different phosphate/polyphosphate ratio in the tribofilm. It also explains why tribofilms form at low temperatures in sliding and rolling-sliding contacts, but not in pure rolling ones where shear stresses within the contact are very low [45].

This mechanism also provides an explanation as to why ZDDPs form similar tribofilms on many different types of surfaces, ranging from metals to ceramics. This is because the primary driver is the stress generated by rubbing a pair of high elastic modulus and hard surfaces together rather than a chemical reaction between ZDDP molecules and surface-derived material. There may be an initial ZDDP molecule-surface interaction that promotes adhesion of the film to the rubbing surfaces, but the subsequent build-up of a thick film is probably an oligomerisation process as suggested by Jones and Coy [46], not involving the substrate.

A shear stress-driven process also explains why ZDDPs forms their characteristic rough, pad-like film structure. In thin-film conditions, ZDDP film formation will begin at asperity conjunctions, where the contact pressure and thus the shear stress is highest. But once the film starts to form on asperities, it will continue grow only at these locations since the developing pads will bear a higher and higher proportion of the applied load. The characteristic deep valleys that separate ZDDP tribofilm pads result simply from there being negligible shear stress present in these regions. This is a relevant insight since the roughness of ZDDP films is believed to be the cause of their giving high friction in mixed lubrication conditions [9].

Another characteristic feature of ZDDP tribofilms is that, at least in simple ZDDP solutions, the thickness of their pads usually levels out at a fixed thickness between 120 and $200 \mathrm{~nm}$. Initially, it was proposed that this originated from a balance between film formation and removal [47], but tests in which a ZDDP tribofilm was formed and then the solution replaced by base oil showed no subsequent ZDDP film removal process [7]. So why does the film thickness level out at a specific value? If film formation is driven by a stress-promoted mechanism, then the film will cease to grow when the shear stress becomes too low to drive the process. This may occur if the pads start to yield in a plastic fashion, thus increasing their contact area. It is well known that a thin layer of a softer material on a harder substrate exhibits a greater effective yield stress under compression than its bulk material due to the constraining effect of the bounding surfaces and that this strength decreases as the layer thickness increases. Bishop et al. [48] and Adams et al. [49] discuss the deformation behaviour of solid cylinders compressed between two rigid platens and suggest a number of expressions to describe the critical mean pressure, $\bar{p}$, required to cause plastic deformation. All of these are based on the aspect ratio of the cylinder height, $h$, to its diameter, $2 r$, for example [49];

$\bar{p}=\sigma_{o}\left[1+\frac{\mu}{3} \frac{2 r}{h}\right]$

where $\mu$ is the coefficient of friction and $\sigma_{o}$ the uniaxial yield stress of the bulk cylinder material. If we consider a typical ZDDP pad with diameter $=5 \mu \mathrm{m}$ and friction coefficient $=0.1$, Eq. 6 suggests that this critical yield pressure may fall to the applied pressure, allowing plastic deformation, when the thickness of ZDDP pads approaches ca $150 \mathrm{~nm}$.

The fact that shear stress controls ZDDP film formation may also explain a phenomenon observed intermittently by the authors that even in thin-film sliding/rolling conditions, solutions of ZDDP in an API Group 3 or group 4 base oil sometimes fail to form tribofilms. This is reported in [50] and can usually be resolved by adding a dispersant to the base oil or replacing the Group 3 or 4 oil by a Group 2 one. It might originate from the very low EHD friction produced by some Group 3 and 4 base oils and a consequently low shear stress even in mixed lubrication conditions.

It is of interest to consider where within the ZDDP molecule the application of a mechanical force might deform or stretch a bond sufficiently to promote the rate of formation of a ZDDP tribofilm. This is not straightforward since the reaction sequence via which ZDDP forms a phosphate film is still poorly understood [1]. One possibility is promotion of breakage of the bond between the alkyl (or aryl) group and $\mathrm{O}$ (or $\mathrm{S}$ after $\mathrm{O} / \mathrm{S}$ exchange [46]). It has long been recognised that secondary alkyl ZDDPs show faster tribofilm formation and lower thermal stability than primary ones, suggesting that strength of this bond influences the rate of ZDDP reaction [7, 51, 52]. However, another simpler possibility is that applied stress acts on the ZDDP molecule to stretch $\mathrm{S}-\mathrm{Zn}$ or $\mathrm{O}-\mathrm{Zn}$ bonds, thus making these more polar and less covalent. This is likely to considerably increase the nucleophilicity of the resulting thiophosphate or phosphate ion, promoting polymerisation [46], and also to reduce the solubility of the molecule.

Since shear stress seems to be an important driver for ZDDP reaction, an interesting conjecture is whether the rate of film formation of a ZDDP may be influenced by the architecture of its alkyl groups via the latters' ability to transmit shear force to the rest of the molecule. In EHD lubrication, branched hydrocarbons tend to give higher EHD friction than linear chain ones since they cannot easily slide past one another during shear [53] and Hoshino et al. [54] found that a di-ethylhexyl-based ZDDP gave faster film formation that a di- $n$-octyl-based one, despite both being primary ZDDPs. It should also be noted that very earliest ZDDP patent was based on cyclic alcohols 
rather than linear or branched chain ones [55] and cyclic hydrocarbons form the basis of almost all traction fluids.

Finally, it is of interest to consider whether a similar stress-promoted thermal activation mechanism is likely to be prevalent with other tribofilm-forming lubricant additives. Certainly, it appears quite probable that the reaction of the friction modifier MoDTC to form low-shear-strength $\mathrm{MoS}_{2}$ nanocrystals is stress-promoted. Like ZDDP, MoDTC is relatively indifferent to the nature of the substrate on which it forms $\mathrm{MoS}_{2}$. Thus, it reduces friction not just on steel but also on pre-formed ZDDP tribofilms [56], ceramics [57] and DLCs [58]. Graham et al. found that the formation of $\mathrm{MoS}_{2}$ from MoDTC and consequent friction reduction was strongly dependent on the severity of the contact conditions [59]. Thus, it occurred rapidly in reciprocated contact conditions with both rough and smooth surfaces but only with rough surfaces in linear sliding conditions. In rolling-sliding conditions, friction reduction only took place at high applied load, not at low load. All of this suggests that a high shear stress produced by a high pressure at asperity contacts is necessary for MoDTC to react. Indeed, it is conceivable that the "synergy" often noted between MoDTC and ZDDP in which ZDDP appears to improve MoDTC's friction-reducing capability [60] may originate from ZDDP forming a rough tribofilm and thus providing regions of enhanced pressure and consequently enhanced shear stress at which MoDTC can react on otherwise smooth surfaces.

It is less likely that tribofilm formation by zinc-free $\mathrm{P}$ and P/S-based antiwear additives is driven predominantly by shear stress. In the absence of $\mathrm{Zn}^{2+}$, these additives require the generation of a suitable metal cation, normally $\mathrm{Fe}^{2+} / \mathrm{Fe}^{3+}$, by rubbing or corrosion, in order to form thick phosphate-based films and this process is likely to be the rate-determining step. It is noteworthy that these additives tend to form tribofilms much more slowly than ZDDP and their film formation is much more sensitive than ZDDP's to the composition of the rubbing solids, with large differences in tribofilm formation even between different steels.

As well as explaining previously poorly understood features of ZDDP tribofilm formation, this confirmation this ZDDP reaction is controlled by the stress-promoted thermal activation reaction model has some significant implications in terms of ZDDP molecular design and use. Thus, it should be possible to tune ZDDP reactivity in a less empirical fashion than in the past and even design ZDDPs for different types of lubricated contact. Clearly, considerable further work still needs to be done; in particular to use a test apparatus that can reach high shear stresses with steel/steel contacts and to study and compare a range of single-component ZDDPs of differing structures, unlike the mixed ZDDP employed in this study. It has been suggested that primary and secondary ZDDPs undergo different reaction routes [52] and this might be expressed in differing shear stress responses. However, the approach outlined in this study, of separating ZDDP reactivity into a thermal activation energy and an activation volume, and thus exploring the effects of both temperature and shear stress on ZDDP tribofilm formation, appears very fruitful.

\section{Conclusions}

This paper shows unambiguously that the shear stress experienced by ZDDP solutions controls the rate at which ZDDP forms a tribofilm on rubbing surfaces and that rate of film formation depends on shear stress in agreement with the stress-promoted thermal activation model. It has been shown that the application of a shear stress can effectively reduce the thermal activation barrier to ZDDP film formation by half. The fact that shear stress drives ZDDP film formation explains many features of ZDDP behaviour; why it forms a tribofilm on very different types of surface, why it forms a film only when sliding contact is present, why the films are rough and why they level out at a critical thickness. The finding also highlights the importance of mechanochemistry to tribology. It suggests that the reactions of other lubricant additives, in particular the soluble organomolybdenum friction modifiers, may be also be best understood in terms of the influence of applied frictional forces on the chemical reactions involved.

The finding that ZDDP reaction is driven by the mechanically applied shear forces suggests ways that the reactivity of ZDDP and also that of other additives may be tuned in an informed manner by molecular design. This is of considerable practical significance as antiwear and friction modifier additives are become increasingly important with the introduction of ever lower-viscosity lubricants. The confirmation of the relevance of the stresspromoted thermal activation model should also prove of value in modelling lubricant additive behaviour and thus helps towards the long sought-after goal of reliable models of boundary lubrication.

Acknowledgement The authors wish to thank Idemitsu Kosan for supply of the high-traction base fluid DM2H for use in this study and Dr. Ksenija Topolovec-Miklozic for adaptation of Fig. 2.

Data Statement All data pertinent to this study is made freely available upon request to the following address; tribology@imperial.ac.uk.

Open Access This article is distributed under the terms of the Creative Commons Attribution 4.0 International License (http://crea tivecommons.org/licenses/by/4.0/), which permits unrestricted use, distribution, and reproduction in any medium, provided you give appropriate credit to the original author(s) and the source, provide a link to the Creative Commons license, and indicate if changes were made. 


\section{References}

1. Spikes, H.A.: The history and mechanisms of ZDDP. Tribol. Lett. 17, 465-485 (2004)

2. Martin, J.M., Grossiord, C., Le Mogne, T., Bec, S., Tonck, A.: The two-layer structure of Zndtp tribofilms: Part I: AES, XPS and XANES analyses. Tribol. Int. 34, 523-530 (2001)

3. Liu, E., Kouame, S.D.: An XPS study on the composition of zinc dialkyl dithiophosphate tribofilms and their effect on camshaft lobe wear. Tribol. Trans. 57, 18-27 (2014)

4. Topolovec-Miklozic, K., Forbus, T.R., Spikes, H.A.: Film thickness and roughness of ZDDP antiwear films. Tribol. Lett. 26, 161-171 (2007)

5. Martin, J.M.: Antiwear mechanisms of zinc dithiophosphate: a chemical hardness approach. Tribol. Lett. 6, 1-8 (1999)

6. Fujita, H., Spikes, H.A.: Formation of zinc dithiophosphate antiwear films. Proc. Inst. Mech. Eng. J. 218, 265-277 (2004)

7. Fujita, H., Glovnea, R.P., Spikes, H.A.: Study of zinc dialkydithiophosphate antiwear film formation and removal processes, part I: experimental. Tribol. Trans. 48, 558-566 (2005)

8. Bancroft, G.M., Kasrai, M., Fuller, M., Yin, Z., Fyfe, K., Tan, K.H.: Mechanisms of tribochemical film formation: stability of tribo-and thermally-generated ZDDP films. Tribol. Lett. 3, 47-51 (1997)

9. Taylor, L., Dratva, A., Spikes, H.A.: Friction and wear behavior of zinc dialkyldithiophosphate additive. Tribol. Trans. 43, 469-479 (2000)

10. Suominen Fuller, M.L., De Jong, K.L., Kasrai, M., Bancroft, G.M.: Electroless generation of phosphate films on metals from zinc dialkyldithiophosphates. Chem. Mater. 12, 1300-1304 (2000)

11. Buckley, D.H.: Auger spectroscopy analysis of lubrication with zinc dialkyldithiophosphate of several metal combinations in sliding contact. NASA-TP-1489 (1979)

12. Mee, M., Torrance, A.A.: A study of the lubricated wear of plain bearing materials in a ZDDP-containing lubricant. Wear 128, 201-217 (1988)

13. Yang, D., Zhou, J., Xue, Q.: Study of the tribochemical behaviour of $\mathrm{Al}$ ion-implanted pure iron lubricated with ZDDP. Surf. Coat. Technol. 102, 223-232 (1998)

14. Sheasby, J.S., Caughlin, T.A., Mackwood, W.A.: A comparison of the boundary lubrication of 52100 steel, zirconia and silicon nitride by $\mathrm{S}, \mathrm{P}, \mathrm{S} / \mathrm{P}$ and zinc dialkyldithiophosphate additives. Wear 196, 100-109 (1996)

15. Mingwu, B., Xushou, Z., Shangkui, Q.: Tribological properties of silicon nitride ceramics coated with molybdenum films under boundary lubrication. Wear 169, 181-187 (1993)

16. Haque, T., Morina, A., Neville, A., Kapadia, R., Arrowsmith, S.: Non-ferrous coating/lubricant interactions in tribological contacts: assessment of tribofilms. Tribol. Int. 40, 1603-1612 (2007)

17. Gosvami, N.N., Bares, J.A., Mangolini, F., Konicek, A.R., Yablon, D.G., Carpick, R.W.: Mechanisms of antiwear tribofilm growth revealed in situ by single-asperity sliding contacts. Science 348, 102-106 (2015)

18. Neville, A., Morina, A., Kapadia, R., Arrowsmith, S., Haque, T.: Study of the ZDDP antiwear tribofilm formed on the DLC coating using AFM and XPS techniques. J. ASTM Int. 4, 1-11 (2007)

19. Yin, Z., Kasrai, M., Fuller, M., Bancroft, G.M., Fyfe, K., Tan, K.H.: Application of soft X-ray absorption spectroscopy in chemical characterization of antiwear films generated by ZDDP Part I: the effects of physical parameters. Wear 202, 172-191 (1997)

20. Jaeger, J.C.: Moving sources of heat and the temperature at sliding contacts. Proc. R. Soc. NSW 56, 203-214 (1942)

21. Mosey, N.J., Müser, M.H., Woo, T.K.: Molecular mechanisms for the functionality of lubricant additives. Science $\mathbf{3 0 7}$, 1612-1615 (2005)
22. Tse, J.S., Song, Y., Liu, Z.: Effects of temperature and pressure on ZDDP. Tribol. Lett. 28, 45-49 (2007)

23. Nakayama, K.: Triboemission of charged particles and resistivity of solids. Tribol. Lett. 6, 37-40 (1999)

24. Kajdas, C.: Importance of the triboemission process for tribochemical reaction. Tribol. Int. 38, 337-353 (2005)

25. Camara, C.G., Escobar, J.V., Hird, J.R., Putterman, S.J.: Correlation between nanosecond X-ray flashes and stick-slip friction in peeling tape. Nature 455, 1089-1092 (2008)

26. Kajdas, C., Tummler, R., von Ardenne, H., Schwarz, W.: The relevance of negative ion mass spectroscopy to the interpretation of the reaction of metal dialkyldithiophosphates during lubricated rubbing. ZFI Mitteilungen 115, 107-112 (1986)

27. Prandtl, L.: Ein Gedankenmodell zur kinetischen Theorie der festen Körper. Z. Angew. Math. Mech. 8, 85-106 (1928)

28. Eyring, H.: Viscosity, plasticity, and diffusion as examples of absolute reaction rates. J. Chem. Phys. 4, 283-291 (1936)

29. Schallamach, A.: The velocity and temperature dependence of rubber friction. Proc. Phys. Soc. B 66, 386-392 (1953)

30. Zhurkov, S.N.: Kinetic concept of the strength of solids. Int. J. Frac. Mech. 1, 311-322 (1965)

31. Bell, G.: Models for the specific adhesion of cells to cells. Science 200, 618-627 (1978)

32. Beyer, M.K., Clausen-Schaumann, H.: Mechanochemistry: the mechanical activation of covalent bonds. Chem. Rev. 105, 2921-2948 (2005)

33. Craig, S.L.: Mechanochemistry: a tour of force. Nature $\mathbf{4 8 7}$, 176-177 (2012)

34. Spikes, H., Tysoe, W.: On the commonality between theoretical models for fluid and solid friction, wear and tribochemistry. Tribol. Lett. 59, 1-14 (2015)

35. Glasstone, S., Laidler, K.J., Eyring, H.: The Theory of Rate Processes. Chapter IX. McGraw-Hill Book Company, New York (1941)

36. Ribas-Arino, J., Marx, D.: Covalent mechanochemistry: theoretical concepts and computational tools with applications to molecular nanomechanics. Chem. Rev. 112, 5412-5487 (2012)

37. Makarov, D.E.: Mechanochemistry of biological and synthetic molecules. J. Chem. Phys. 144, 030901 (2016)

38. Ghanbarzadeh, A., Parsaeian, P., Morina, A., Wilson, M.C., van Eijk, M.C., Nedelcu, I., Dowson, D., Neville, A.: A semi-deterministic wear model considering the effect of zinc dialkyl dithiophosphate tribofilm. Tribol. Lett. 61, 1 (2016)

39. Felts, J.R., Oyer, A.J., Hernández, S.C., Whitener Jr, K.E., Robinson, J.T., Walton, S.G., Sheehan, P.E.: Direct mechanochemical cleavage of functional groups from graphene. Nat. Commun. 6, 6467 (2015)

40. Adams, H.L., Garvey, M.T., Ramasamy, U.S., Ye, Z., Martini, A., Tysoe, W.T.: Shear-induced mechanochemistry: pushing molecules around. J. Phys. Chem. C 119, 7115-7123 (2015)

41. Johnson, K.L.: Contact Mechanics. Cambridge University Press, Cambridge (1985)

42. Goda, T.: The latest trend in traction fluids. Idemitsu Tribo Rev. 34(21), 11-14 (2011)

43. Hamrock, B.J., Dowson, D.: Ball Bearing Lubrication. The Elastohydrodynamics of Elliptical Contacts. Wiley, NY (1981)

44. Archard, J.F.: The temperature of rubbing surfaces. Wear 2, 438-455 (1959)

45. Gunsel, S., Spikes, H.A., Aderin, M.: In-situ measurement of ZDDP films in concentrated contacts. Tribol. Trans. 36, 276-282 (1993)

46. Jones, R.B., Coy, R.C.: The chemistry of the thermal degradation of zinc dialkyldithiophosphate additives. ASLE Trans. 24, 91-97 (1981)

47. So, H., Lin, Y.C.: The theory of antiwear for ZDDP at elevated temperature in boundary lubrication condition. Wear $\mathbf{1 7 7}$, 105-115 (1994) 
48. Bishop, J.F.W.: On the effect of friction on compression and indentation between flat dies. J. Mech. Phys. Solids 6, 132-144 (1958)

49. Adams, M.J., Edmondson, B., Caughey, D.G., Yahya, R.: An experimental and theoretical study of the squeeze-film deformation and flow of elastoplastic fluids. J. Non-Newton. Fluid Mech. 51, 61-78 (1994)

50. Benedet, J.F.L.: Low and zero SAPs antiwear additives for engine oils; Chapter 5. Ph.D. Thesis Imperial College London (2012)

51. Bennett, P.A.: A surface effect associated with the use of oils containing zinc dialkyl dithiophosphate. ASLE Trans. 2, 78-90 (1959)

52. Mosey, N.J., Woo, T.K.: A quantum chemical study of the unimolecular decomposition mechanisms of zinc dialkyldithiophosphate antiwear additives. J. Phys. Chem. A 108, 6001-6016 (2004)

53. Hentschel, K-H.: The influence of molecular structure on the frictional behaviour of lubricating fluids. J. Synth. Lubr. 2, 143-174, 238-160 (1985)

54. Hoshino, K., Yagashita, K., Tagawa, K., Spikes, H.A.: Tribological properties of sulphur-free antiwear additives zinc dialkylphosphates (ZDPs). SAE Int. J. Fuels Lubr. 5, 504-510 (2012)

55. Asseff, P.A.: Lubricant. US Patent 2261047 (1941)

56. Topolovec-Miklozic, K., Forbus, T.R., Spikes, H.A.: Performance of friction modifiers on ZDDP-generated surfaces. Tribol. Trans. 50, 328-335 (2007)

57. Yajun, M., Wancheng, Z., Shenghua, L., Yuansheng, J., Yucong, W., Simon, T.: Tribological performance of three advanced piston rings in the presence of MoDTC-modified GF-3 oils. Tribol. Lett. 18, 75-83 (2005)

58. Topolovec-Miklozic, K., Lockwood, F., Spikes, H.: Behaviour of boundary lubricating additives on DLC coatings. Wear $\mathbf{2 6 5}$, 1893-1901 (2008)

59. Graham, J., Korcek, S., Spikes, H.A.: The friction-reducing properties of molybdenum dialkyldithiocarbamate additives. Part 1. Factors influencing friction reduction. Tribol. Trans. 44, 626-636 (2001)

60. Spikes, H.A.: Friction modifier additives. Tribol. Lett. 60, 5 (2015) 